\title{
Os avanços tecnológicos do século XXI e o desenvolvimento de habilidades necessárias ao profissional do Direito a partir das abordagens das Universidades de Harvard e Stanford
}

\author{
The technological advances of the XXI century and the development of necessary \\ skills for the professional of law by Havard's and Standford's apprroaches
}

\author{
Lilia Maia de Morais Sales* \\ Mário Quesado Miranda Bezerra*
}

\section{Resumo}

\begin{abstract}
No século $X X I$, houve um considerado avanço tecnológico. Desse modo, observam-se reflexos dessas inovações no mercado de trabalho, em especial em relação à atuação do profissional da ciência jurídica. Assim, este trabalho se propõe a analisar quais são as perspectivas do mercado de trabalho à luz dessas inovações; apresentar as competências e habilidades necessárias para a atualização do profissional do Direito, de modo a se manter atuante nesse cenário; e, por fim, analisar como escolas de Direito americanas vêm promovendo o desenvolvimento dessas capacidades. Conclui-se pela mudança das matrizes curriculares das instituições de ensino jurídico, em prol de uma atualização pertinente ao exigido pela nova conjuntura do mercado.
\end{abstract}

Palavras-chave: Habilidades do século XXI. Inovação jurídica. Mercado de trabalho.

\section{Abstract}

In the 21st century, there was a considered technological advance. In this way, it can be observed the reflexes of these innovations in the labor market, especially the one related to the work of the legal science professional. Thus, this paper proposes to analyze the perspectives of the labor market in the light of these innovations; to present what are the skills and abilities necessary for the updating of the legal professional in order to remain active in this scenario; Finally, to analyze how American law schools have been promoting the development of these capabilities. It concludes by changing the curricular matrices of the legal education institutions in order to update to the one required by the new market environment.

Keywords: $21^{\text {st }}$ century abilities. Legal innovation. Labor market.

\section{Introdução}

Com o desenvolvimento das tecnologias nos mais variados ramos, desde a manipulação genética até a transmissão de informações, faz-se relevante observar a necessidade de adaptação das competências requeridas por essa evolução, nas mais diversas profissões, uma vez que o impacto causado por aquelas no mercado de trabalho exigirá de seus profissionais novas habilidades. A Universidade de Stanford, em seu encontro New Breakthroughs in Computational Law - Code X Future Law Conference 2015, explanou que, apesar da grande capacidade da inteligência artificial na substituição do trabalho humano em muitas áreas do conhecimento, a intervenção do ser humano se faz necessária, em especial, em situações que envolvem procedimentos cognitivos e relações que tangenciem processos emocionais (VERJEIJL, 2015).

Doutora pela Universidade Federal de Pernambuco. Formação em mediação de conflitos na Universidade de Harvard, junto ao Program on Negotiation (EUA). Advogada, mediadora, professora titular da Universidade de Fortaleza no Programa de Pós-Graduação em Direito Constitucional e no Mestrado Profissional em Direito e Gestão de Conflitos. Vice-Reitora de Pós-Graduação da Universidade de Fortaleza e coordenadora de vários projetos de pesquisa com foco no estudo da mediação de conflitos. Bolsista de Produtividade em Pesquisa - CNPq. Fortaleza - CE - Brasil. E-mail: lilia@unifor.br.

Mestre em Direito Constitucional das relações privadas pela Universidade de Fortaleza. Professor na Faculdade Cearense - FaC. Fortaleza - CE - Brasil. E-mail: mario_quesado@hotmail.com. 
A área jurídica é apontada como a seara profissional que terá forte impacto da tecnologia, sendo necessário rever as habilidades atuais desenvolvidas pelos profissionais. Assim, o escopo deste artigo é perscrutar, a partir dessa realidade, quais são as inteligências e habilidades requeridas para o profissional jurídico do século XXI. Para tanto, realizou-se levantamento bibliográfico e documental, analisando-se pesquisas e experiências inovadoras que oferecem caminhos para identificar competências que devem ser estimuladas na formação atual na área jurídica.

\section{Perspectivas globais acerca do mercado de trabalho no século XXI}

A expansão da tecnologia nas mais variadas áreas do conhecimento, nas palavras de Schwab (2016), coloca o mundo diante da Quarta Revolução Industrial, caracterizada por: inteligência artificial, internet das coisas, armazenamento de energia, biotecnologia, veículos autônomos e as mais variadas inovações. Nos últimos 20 anos, consequentemente, houve uma modernização do espaço de exercício das atividades laborais e um aumento na demanda de pessoal capacitado para atuar nesse meio (BALCAR, 2014; CARNEVALE, 2013; EGER; GROSSMANN, 2004; INTERNATIONAL LABOUR ORGANIZATION, 2008).

No ano de 2015, o World Economic Forum (WEF) realizou uma pesquisa ${ }^{1}$ para averiguar quais seriam as habilidades necessárias para o mercado de trabalho do século XXI (Figura 1), mediante a expansão dessas tecnologias, e quais as perspectivas até o ano de 2020. Combinadas com a atual revolução tecnológica, o que aparece como habilidade para os profissionais do século XXI são habilidades voltadas para o humano, isto é, para a capacidade intrínseca do ser humano de reflexão, empatia, vivência de emoções, análise e tomada de decisões, conhecidas como soft skills.

A solução de problemas complexos aparece como habilidade principal, seguida do pensamento crítico, da criatividade, do gerenciamento de pessoas, da coordenação com outros (horizontalidade nas relações), da inteligência emocional, da capacidade de tomada de decisões, da orientação de serviços e da negociação e flexibilidade cognitiva, que são as proficiências necessárias ao profissional do século XXI.

Nesse diapasão, Susskind e Susskind (2017, p. 117) compreendem que, embora as máquinas possuam capacidades físicas (movimentos mais velozes e levantamento de cargas mais pesadas), há de se destacar o domínio do ser humano sobre certos tipos de tarefa, em especial aquelas correlacionadas ao uso das capacidades perceptivas, emocionais e cerebrais. ${ }^{2}$

Em uma meta-análise, ${ }^{3}$ o Fórum Mundial de Economia, com o escopo de identificar, em primeira instância, quais seriam as habilidades necessárias para atender às necessidades do mercado de trabalho do século XXI, dividiu as habilidades em três categorias: instrução fundamental; competências e qualidades de caráter (WEF, 2015).

Instrução fundamental diz respeito à maneira como o indivíduo aplica as habilidades centrais no cotidiano. Essas habilidades correspondem ao ponto de partida para o pleno domínio daquelas necessárias ao século

\footnotetext{
A pesquisa realizada teve por objetivo compreender quais as possíveis expectativas com relação ao futuro dos trabalhos, empregos e habilidades dos maiores empregadores das maiores economias do mundo. A pesquisa teve como ponto de partida focar nos chefes dos departamentos de recursos humanos. Foram analisadas indústrias de diversos setores, tais como de Infraestrutura (Química, Planejamento Urbano e Mineração); Consumo (Alimentos, Produção de Bens, Agricultura); Energia (Energias renováveis, Combustíveis e Utilidades e Tecnologias das energias); Serviços de Investimento e Financeiro (Bancos, Mercado de Capitais, Investidores Privados); Saúde (Planos de Saúde e Saúde Global); Informação (Tecnologia da Informação e Comunicação); Mídia (Mídia, Entretenimento e Informação); Mobilidade (Aviação e viagens, Automóveis e Meios de Transporte); Serviços Profissionais (profissionais liberais).

Os países analisados foram divididos por região: Ásia e Pacífico (Austrália, China, Indonésia, Índia e Japão); Europa e Ásia Central (França, Alemanha, Itália, Turquia e Reino Unido); Oriente Médio e Norte da África (Conselho de Cooperação do Golfo); África Subsaariana (África do Sul); Américas (Brasil, México e Estados Unidos). WORLD ECONOMIC FORUM. Future of Jobs report. (2016, p. 57).

No original: We can accept that machines can move quicker and lift heavier, but we like to think we hold domination over certain types of tasks, especially those that require brainpower and emotion.

A pesquisa utilizou como base os trabalhos elaborados pela European Skills, Competences, Qualifications and Occupations (ESCO), Parcerias para as habilidades do século 21, em Gauge, Brookings e Pearson.
} 
XXI. Estão inclusas neste ponto não somente a capacidade de leitura (alfabetização) e de conhecimento numérico, mas também o entendimento acerca das ciências, do domínio tecnológico e informacional, do financeiro, do cultural e do legislativo (WEF, 2015). Corrobora esse pensamento a American Managment Association e Partnership for 21st Century Skills (2010, online):

Executivos dizem necessitar de uma força de trabalho prontamente equipada com habilidades que vão além das básicas de ler, escrever e conhecimento aritmético para crescer em seus negócios. Capacidades como o pensamento critico, a comunicação, a colaboração e a criatividades serão de maior importância para as organizações empresárias no futuro. (Traduziu-se) ${ }^{4}$

As competências correspondem à forma como o sujeito aborda o problema complexo com intuito de resolver a demanda, e englobam a criatividade, isto é, o uso da imaginação como ferramenta para a criação de jeitos inovadores de interpelar o problema; o pensamento crítico, que corresponde à capacidade de identificar, avaliar e analisar situações de forma a propor respostas aos problemas; a comunicação, manifestada a partir da maneira como um indivíduo se faz compreender, e é capaz de entender aquilo que Ihe foi dito; e a colaboração, que corresponde à habilidade de desenvolver atividades em equipe e unificar forças de trabalho e inteligências $\left(\mathrm{WEF}^{5}, 2015\right)$.

Por fim, entende-se por qualidades de caráter o modo de agir do indivíduo perante um ambiente mutável. Neste ponto, a persistência e a adaptabilidade são habilidades cujas funções dizem respeito à resiliência que deve ser empregada quando em face de uma dificuldade; iniciativa e curiosidade, que são os pontos de ignição para a descoberta de novos conceitos e ideias; e liderança e consciência social e cultural, que são situações que envolvem interações construtivas com outros, de sorte que essas ocorrem de maneira respeitosa nos aspectos sociais, éticos e culturais (WEF, 2015).

Figura1

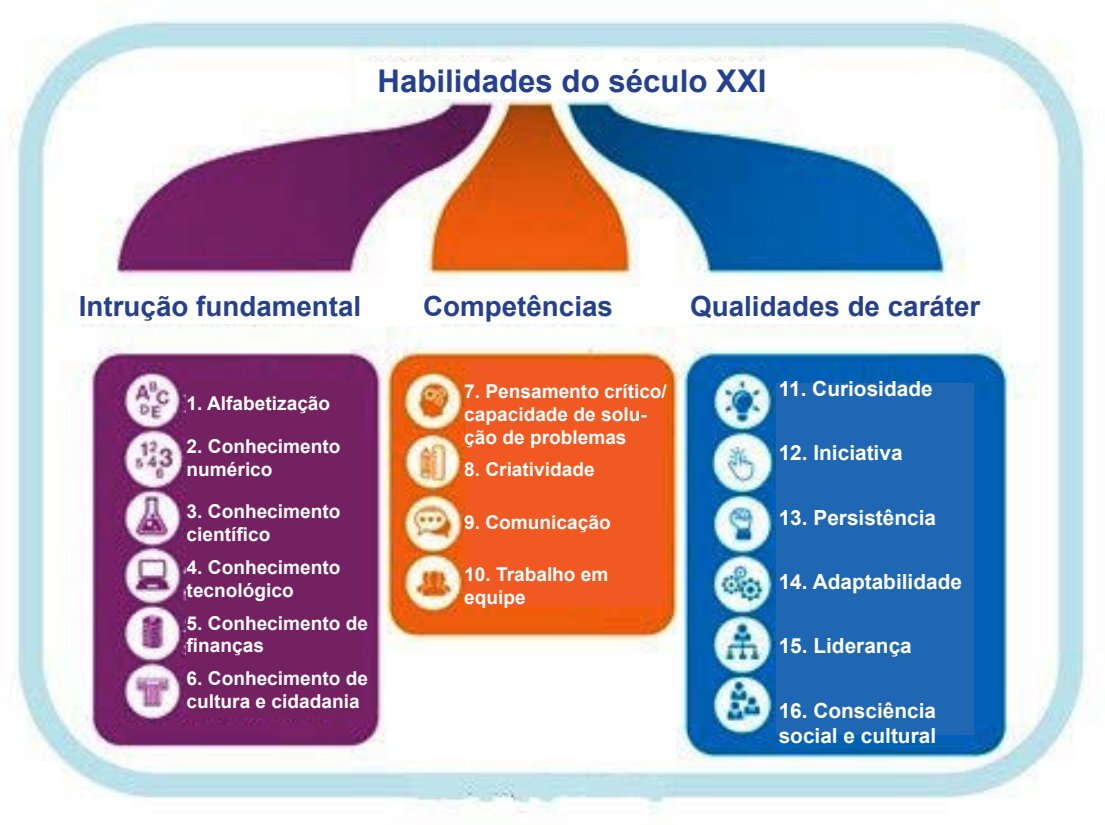

Fonte: World Economic Forum (2015).

Em 2018, o Fórum Econômico Mundial reforça as pesquisas de 2015 e apresenta, novamente, a importância do desenvolvimento das habilidades humanas de criar, resolver problemas e tomar decisões,

\footnotetext{
No original: Executives say they need a workforce fully equipped with skills beyond the basics of reading, writing and arithmetic (the "3 Rs") to grow their businesses. Skills such as critical thinking, communication, collaboration, and creativity (the 4 Cs) will become even more important to organizations in the future.

5 WORLD ECONOMIC FORUM. New Vision for Education - Unlocking the Potential of Technology, 2015.
} 
relatando que atividades de conhecimento repetitivo ou rotineiro serão automatizadas e que o desenvolvimento das habilidades apontadas pode abrir uma nova gama de oportunidades (WEF, 2018). Assim, lideranças ${ }^{6}$ alertam que deve haver uma mudança na forma de educação, visto o eminente perigo de ser deixado no passado. Põe-se em destaque o aprendizado de habilidades como o trabalho em equipe, a consciência dos valores e do pensamento crítico-criativo e a iminência da substituição de trabalhos manuais, rotineiros e repetitivos por autômatos (WEF, 2018).

Seguindo esses resultados, o McKinsey Global Institute apresenta pesquisa informando que, em função do avanço tecnológico, o mercado de trabalho sofrerá impactos no sentido de que, até 2030, haverá substituição de cerca de 800 (oitocentos) milhões de trabalhadores humanos por tecnologias de inteligência artificial (MCKINSEY GLOBAL INSTITUTE, 2017).

A Deloitte apresentou relatório, em 2016, apontando que o conhecimento técnico, adquirido pelos estudantes em suas formações acadêmicas representa apenas uma fração das competências e habilidades essenciais ao mercado de trabalho. A aquisição e desenvolvimento de outros talentos, tais como o essencial cognitivo, a habilidade de entrosamento e sociabilidade e a capacidade de solução de problemas, além do know how ${ }^{7}$ de como aplicar tais competências em situações concretas, mostram-se relevantes no contexto de empregabilidade. O referido relatório ainda apresenta dados no sentido da capitalização de lucro que o desenvolvimento dessas habilidades proporciona:

Um aumento de 10\% na importância das habilidades cognitivas, que influenciam na aquisição e aplicação de conhecimento, contribui para um aumento de $12 \%$ na média dos ganhos por hora trabalhada. O mesmo aumento na importância das habilidades sociais contribui para um aumento de $10 \%$ na hora média trabalhada (Traduziu-se). ${ }^{8}$

Com a evolução tecnológica e o desenvolvimento de economias fundadas em inovação, criatividade e colaboração, o mercado de trabalho sofreu impactos, havendo declínio na necessidade de operadores que realizassem trabalhos de cunho manual ou cognitivo, porém notou-se elevada e correspondente demanda naqueles que careciam de competências de rotinas não analíticas e habilidades interpessoais.

Com a mudança do eixo de competências, foi diagnosticado o problema da provisão de agentes capacitados com tais habilidades. Como exemplo disso, o Manpower Group (2014) evidenciou a dificuldade de contratação ocorrida por conta da ausência de pessoal qualificado, visto que apenas $6 \%$ dos colaboradores eram capazes de solucionar problemas com proficiência em ambientes tecnologicamente ricos.

Dentre as habilidades necessárias, as apontadas como mais importantes foram: capacidade de solucionar problemas complexos; liderança; comunicação; entendimento de tecnologia; trabalho em equipe e gerenciamento de pessoas; pensamento criativo; gerenciamento de projetos; e conhecimento funcional (ACCENTURE, 2013).

Dessa forma, a Accenture (2013) identificou estratégias capazes de promover uma diminuição desse lapso, quais sejam: a busca pelo equilíbrio entre conhecimento formal e informal; englobamento de novas formas de desenvolver habilidades; expansão das opções de candidatos; percepção de talentos, por meio de novas tecnologias; e investimento em talento no começo da vida acadêmica.

Apresenta como estratégia de fortalecimento dessas habilidades o estímulo de parcerias com instituições de ensino superior, no intuito de revisar as matrizes curriculares e propor formas de incentivar as habilidades dos estudantes, de forma que possam adquirir e desenvolver essas capacidades ao longo de seu programa de graduação (ACCENTURE, 2013).

Diante do exposto, ressalta-se a relevância do desenvolvimento de competências, de modo a adequar o profissional do século XXI à realidade na qual se encontra inserido. Portanto, cabe a quebra do paradigma

Exemplo dos entrevistados: Jack Ma, fundador da Alibaba, maior comerciante eletrônico da China; Minouche Shafik, diretora da Escola de Economia de Londres; Fabiola Gianotti, diretora geral da CERN; Justin Trudeau, primeiro ministro do Canadá.

N.T. Saber fazer.

8 A ten per cent increase in the importance of cognitive abilities, which influence the acquisition and application of knowledge, contributes to a 12 per cent increase in median hourly earnings. The same increase in the importance of social skills contributes to a ten per cent increase in median hourly earnings. 
da estagnação, do sentimento de que somente o conhecimento técnico é significado de sucesso no mercado de trabalho, uma vez que o desenvolvimento tecnológico já consegue suprir a atividade humana nesse sentido. Dessa forma, faz-se oportuno notar quais são as capacidades que o desenvolvimento eletrônico não consegue suprir, tais como empatia, liderança, criatividade e trabalho em equipe, procurando métodos e formas de estimular o desenvolvimento dessas habilidades.

\section{A perspectiva da atuação do profissional do Direito a partir da evolução tecnológica do século XXI}

Em face dos avanços tecnológicos, o processo de adaptabilidade do profissional da ciência jurídica se mostra em eminente destaque. A mudança no cenário de operações relativas à ciência do Direito, de acordo com Susskind (2013, p. XIII), mostrar-se-á mais expressiva nas próximas duas décadas do que se comparada ao ocorrido nos últimos dois séculos. Em países como a Inglaterra e a Austrália, o monopólio da atividade jurídica não mais é exercido por advogados, visto que profissionais, não necessariamente com formação em Direito, podem conduzir escritórios jurídicos e que as firmas de advogados podem ter ações nas bolsas de valores ou ter financiamento externo, a exemplo de private equity. ${ }^{9}$ Como exemplos, tem-se o Co-Op Bank da Inglaterra, a BT Group, empresa de telecomunicações inglesa, e a Automobile Association, relacionada a veículos e transporte na Austrália, que oferecem serviços jurídicos.

Ainda nesse sentido, o relatório da Deloitte (2016) teve por estimativa que 39\% dos trabalhos que envolvem serviços legais entrarão em risco de extinção, tendo em vista a velocidade de desenvolvimento tecnológico, visto que, segundo Sperandio (2016, p. 6), haveria uma redução de custos decorrente da divisão de funções por tarefa, de forma que atividades como revisão documental e due diligence podem ser terceirizadas.

Nesse sentido, a tendência mundial é de que os escritórios de advocacia passem a exercer serviços voltados não mais somente às práticas legais, mas que integrem ao seu repertório conhecimentos de indústria, comércio, tecnologia da informação e outras áreas de expertise (DELOITTE, 2016).

Em consonância, outro estudo realizado pela mesma instituição concluiu que, no prazo até 2030, menor será a quantidade de escritórios tradicionais de advocacia, devido ao crescimento das funções não jurídicas e ao maior uso da tecnologia como alternativa de carreira; uma nova gama de habilidades dos advogados capazes de se adaptar e promover mudanças às novas necessidades terá maior desenvolvimento; o aumento da flexibilidade e da mobilidade, dada a expansão internacional e a superação de barreiras, como também arranjos de trabalho mais céleres e novos tipos contratuais; e a reformulação da estrutura da força laboral, que permite a captação de funcionários com habilidades dos mais diversificados tipos (DELOITTE, 2016).

Segundo dados levantados por Altman Weil (2015), no que diz respeito aos esforços para aumentar a produtividade e a eficiência do atendimento jurídico, $58 \%$ dos entrevistados ${ }^{10}$ adotaram como medida a substituição de recursos humanos por ferramentas tecnológicas.

Dessa maneira, apresentam-se as Legaltechs (também conhecidas como lawtechs), que consistem em start-ups de soluções tecnológicas voltadas à reformulação do setor jurídico. O termo tem derivação das palavras inglesas Legal/Law ${ }^{11}$ e Technology, ${ }^{12}$ e tem como escopo a colaboração da tecnologia na rotina jurídica. Tal facilidade é promovida mediante a coleta, a leitura, o armazenamento de informações, a unificação dessas informações em bancos de dados, além da capacidade de reproduzir padrões de documentos e a emissão de alertas relativos aos prazos (SALOMÃO, 2017). No entendimento de Praduroux, Paiva e Caro (2016, online), esse tipo de informática jurídica:

\footnotetext{
Essas mudanças foram autorizadas na Inglaterra e no país de Gales mediante o Legal Services Act 2007, que se encontra em vigor desde 2011. Para a realização da pesquisa, foram entrevistados Administradores e Chefes de 797 escritórios de advocacia nos Estados Unidos com 50 funcionários ou mais.

11 Legal/Lei.

12 Tecnologia.
} 
[...] diz respeito às implicações sociais do uso da informática no campo do direito, como armazenamento e obtenção automática de fontes da lei, automação de escritórios de advocacia e administrativo judicial e todos os usos de computadores na lei. (Base de dados; sistema de informação; programas educacionais; sistemas de expertise; redação de texto jurídico auxiliada por computadores). ${ }^{13}$ (Traduziu-se).

Os supramencionados autores, com embasamento no explanado pela Universidade de Harvard, ${ }^{14}$ didaticamente dividiram as formas de lawtechs em oito tipos, quais sejam: mercado jurídico; montagem e automação de documentos; prática gerencial; pesquisa legal; análise preditiva e levantamento de dados de litígios; descoberta eletrônica; resolução de disputas online; e tecnologia de segurança. ${ }^{15}$

Mercado jurídico corresponde ao ambiente digital capaz de promover conexão entre advogados e clientes, finais ou não, e a comunicação com outros advogados (PRADUROUX; PAIVA; CARO, 2016).

Montagem e automação de documentos consiste na categoria que abrange o design de sistemas e fluxos de trabalho que auxiliam na criação de documentos eletrônicos. Estes incluem sistemas baseados em lógica, que utilizam segmentos de texto e/ou dados pré-existentes para reunir um novo documento (PRADUROUX; PAIVA; CARO, 2016).

Prática gerencial diz respeito a um software de gerenciamento de casos e atividades, que fornece aos advogados modos convenientes para um gerenciamento efetivo de informações pertinentes a clientes e casos, tais como contatos, reuniões, documentos e especificidades gerais. Tudo o que está envolvido na facilitação da automação nas práticas de Direito pode ser considerado prática/caso software de gerenciamento. A maioria dos pacotes de programas oferece: gerenciador de casos; registro de monitoramento de tempo e gerenciamento de contatos (PRADUROUX; PAIVA; CARO, 2016).

Pesquisa legal tem por embasamento ferramentas de buscas jurídicas avançadas, capazes de permear os campos da inteligência artificial, da coleta de dados e do processo linguístico natural, de forma a identificar os diferentes recursos e características disponíveis (PRADUROUX; PAIVA; CARO, 2016).

Análise preditiva e levantamento de dados de litígios condizem com a análise de informações e dados por meio de estatística ou de técnicas matemáticas que resultam em uma correlação entre o apurado. Esses resultados são usados para melhor prever situações e a tomada de decisão. A modelagem preditiva de gerenciamento de demandas proporciona informações no começo da litigância com o escopo de apresentar a melhor saída possível (PRADUROUX; PAIVA; CARO, 2016).

A descoberta eletrônica compete ao aspecto de identificar, coletar e produzir informações armazenadas eletronicamente em resposta à produção de provas em um processo ou investigação. Esses dados podem ser exemplificados como correios eletrônicos, documentos, bases de dados, correios de voz e arquivos de texto e vídeo (PRADUROUX; PAIVA; CARO, 2016).

A resolução de disputas online utiliza a tecnologia da internet para propor soluções extrajudiciais de conflitos. Existem duas ramificações dessa forma de legaltech: a primeira, na qual a tecnologia opera de forma ativa na resolução do litígio, a exemplo do sistema de aposta às cegas, que faz uso de variáveis matemáticas no intuito de propor às partes o melhor resultado possível; a segunda consiste em formas de resolução de conflitos auxiliadas pela tecnologia, com o intuito de aumentar o número de processos alternativos de solução de controvérsias, independentemente da tecnologia (PRADUROUX; PAIVA; CARO, 2016).

13 No original: "[...] Legal informatics (legal information technology and its study) is concerned with the social implications of informatics use, as well as with all the applications of informatics in the field of law, such as the storage and the automatic retrieval of sources of law, the automation in law offices and in the judicial administration and all the other uses of the computers in law (data bases; information systems; educational programs; expert systems, computer-aided legal drafting, etc.)". Diz respeito às implicações sociais do uso da informática no campo do direito, como armazenamento e obtenção automática de fontes da lei, automação de escritórios de advocacia e administrativo judicial e todos os usos de computadores na lei (Base de dados; sistema de informação; programas educacionais; sistemas de expertise; redação de texto jurídico auxiliada por computadores).

14 Disponível em: < http://techindex.law.stanford.edu/>. Acesso em: 31 jan 2018.

15 No original: Legal Market Place; Document Automation and Assembly; Practice Management; Legal Research; Predictive Analytics and Litigation Data Mining; Electronic discovery; Online dispute resolution; Data security technologies. 
Por fim, tecnologia de segurança de informações tem por escopo a proteção da confidencialidade dos dados trocados entre servidor e cliente. Para o uso dessas inovações, é preciso de algoritmos e criptografia (PRADUROUX; PAIVA; CARO, 2016).

O mercado possui algumas ferramentas exemplificativas de legaltechs, tais como Contract Express e Exari, que geram documentos jurídicos de alta qualidade após interação com o usuário; o aplicativo Docracy, capaz de selecionar decisões e acórdãos; e o Shake, apto a produzir contratos mediante celulares. Outro instrumento de inovação tecnológica na atividade jurídica são as salas de negociação online, que se constituem plataformas sitiadas na internet, cuja finalidade é propiciar o estímulo à negociação e à mediação antes da judicialização da demanda e a colaboração entre escritório de advocacia e cliente para o compartilhamento e armazenamento de documentos (SUSSKIND; SUSSKIND, 2017).

Ainda como reflexo dessas tecnologias, existem as comunidades online, a exemplo de Legal OnRamp, na qual pessoas, formadas em Direito ou não, contribuem e compartilham suas experiências na solução de disputas judiciais (SUSSKIND; SUSSKIND, 2017).

Ademais, o escritório americano Baker \& Hostetler adquiriu o sistema de inteligência artificial ROSS, que utiliza a tecnologia WATSON da IBM. Constam como habilidades do sistema mencionado: a capacidade de detalhamento sobre ações jurídicas; monitoramento das jurisprudências; solicitação de memorandum para melhor compreensão de problemas legais ou conferência do trabalho em outra fonte de pesquisa; extração de citações e palavras-chave da lei de múltiplos casos, para uma compreensão e análise legal da situação em questão; identificação do embasamento legal para problemas falimentares, com base em decisões de cortes federais e estaduais; identificação de problemas jurídicos de propriedade intelectual, não somente dos tribunais, mas também das decisões administrativas pelas instituições de registros de Marcas e Patentes; e designação de respostas para os casos envolvendo litígios trabalhistas (ROSS, 2018).

Desse modo, nota-se que o profissional jurídico acaba por necessitar do desenvolvimento de habilidades e de certa adaptabilidade a esse novo cenário do mercado de trabalho, tendo em vista a busca por soluções mais céleres, menos conflituosas e por ferramentas e aplicativos capazes de apresentar os mesmos conhecimentos técnicos. Assim, resta ao operador do Direito trabalhar para a expansão de competências as quais as inteligências artificiais não conseguem reproduzir.

\section{Abordagens das instituições internacionais de ensino jurídico em face das inovações do século XXI}

Após demonstrar a gama de competências exigidas ao profissional da ciência jurídica pelos impactos trazidos em decorrência dos avanços tecnológicos do século XXI, cabe a esta pesquisa examinar as metodologias empregadas por instituições de ensino superior no tocante à promoção e ao desenvolvimento dessas habilidades. Para tanto, elegeu-se como foco de abordagem as seguintes instituições internacionais: Universidade de Harvard e Universidade de Stanford, ranqueadas entre as cinco melhores escolas de Direito do mundo. ${ }^{16}$

\subsection{Universidade de Harvard}

É escopo do programa da Escola de Direito de Harvard a promoção de métodos diferenciais que capacitem os graduandos a não serem mais meros repetidores de informação, quebrando o molde de ensino, de maneira a desenvolver formas inovadoras de solução de conflito e equipar o futuro operador do Direito com habilidades essenciais ao século XXI, as quais não podem ser ensinadas pelo meio tradicional de ensino (COLINS, 2017).

Um dos programas utilizados pela referida instituição consiste no Law Without Walls, ${ }^{17}$ cuja finalidade é o treinamento de operadores jurídicos aptos a resolver conflitos de forma criativa e inovadora, de modo a

16 Segundo a QS University Rankings https://www.topuniversities.com/university-rankings/university-subject-rankings/2018/law-legal-studies. 
compreender as necessidades de seus clientes de forma empática; líderes com aptidões multidisciplinares, com tolerância a situações de alto risco e capazes de desenvolver trabalhos em equipe plurais (culturas, países e disciplinas); além de especialistas em situações negociais, competentes no uso de tecnologias e mídias sociais, com enfoque na comunicação e colaboração (COLINS, 2017).

Oficina de Solução de Conflito ${ }^{18}$ visa conectar o estudo teórico da ciência do Direito à prática advocatícia, assim o aluno é apresentado a questionamentos, no intuito de refletir sobre quais os tipos de problemas que advogados resolvem e como o fazem, além de como se dá a construção intelectual e quais os efeitos práticos das conclusões obtidas. Destarte, o discente aprende a interligar o conhecimento jurídico e a parte prática do exercício profissional, no intuito de atingir os objetivos dos clientes dentro dos limites legais. Um dos pontos-chave do workshop é o esforço colaborativo, o que estimula a habilidade do trabalho em grupo, além do tocante à capacidade de liderança (HARVARD, 2018).

O Programa de Pesquisa e Escrita Jurídica ${ }^{19}$ tem como premissa não somente fornecer ao estudante ferramentas para a composição de peças jurídicas, mas fazer com que ele compreenda o problema interno a ser estudado e saiba externar a situação mediante argumentações concisas e pertinentes, assim tendo capacidade de analisar criticamente a situação em prol de obter a melhor solução possível. Desenvolve-se, neste programa, a capacidade de pensamento crítico, a análise cognitiva de informações, a comunicação e a compreensão de posições (HARVARD, 2018).

Nos anos subsequentes, compete ao estudante a eleição de matérias de interesse para completar o número de créditos exigidos para a graduação, de tal sorte que a instituição disponibiliza diversos grupos de estudo com áreas de concentração variadas. Tangentes às habilidades do século 21 , cabe destacar os programas de Direito e Negócios ${ }^{20}$ e Direito, Ciência e Tecnologia ${ }^{21}$ (HARVARD, 2018).

No relativo ao Programa de Direito e Negócios, o aluno compreende a interseção entre a atividade jurídica e a dinâmica das relações negociais. Como exemplo de desenvolvimento de habilidades para tanto, existem as disciplinas de Métodos de Análise para Advogados ${ }^{22}$ e de Estratégias Negociais para Advogados $^{23}$ (HARVARD, 2018).

A primeira matéria tem por meta fazer com que o estudante assimile noções básicas de gestão, negociação e economia. Tal compreensão é estimulada mediante o desenvolvimento de atividades capazes de instruir o discente a possuir organização cognitiva para a tomada consciente de decisão na solução de conflitos (HARVARD, 2018).

Ao tratar das noções de estratégia negocial, a instituição preconiza o fomento ao pensamento analítico de situações concretas, por meio de abordagens econômicas e de teoria dos jogos, aos estudos negociais nos processos de associação, de forma a apresentar suas vantagens e como estruturar situações de colaboração e liderança (HARVARD, 2018).

O grupo de estudos ainda conta com a possibilidade de integração multidisciplinar, no intuito de estimular o engajamento do discente em disciplinas, como a de Liderança Inovadora ${ }^{24}$ da Escola de Negócios de Harvard, que objetiva o pensamento de criação no contexto de inovações industriais. Dessa maneira, promove a competência da criatividade, visto que fomenta no aluno a capacidade de consultoria jurídica nessa seara (HARVARD, 2018).

O Programa de Direito, Ciência e Tecnologia alinha as expertises jurídicas ao cenário de inovação tecnológica nos seus mais variados aspectos, tais como a propriedade industrial, biotecnologia, bioética,

\footnotetext{
Criado pela professora Michele DeStefano, professora da Universidade de Miami e membro convidado do corpo docente do Programa de Educação Executiva da Escola de Direito de Harvard.

No original: Problem Solving Workshop.

No original: Legal Research and Writing Program.

No original: Law and Business.

No original: Law, Science and Technology.

No original: Analytical Methods for Lawyers.

No original: Business Strategies for Lawyers.

No original: Leading Innovative Ventures.
} 
direitos da internet e tecnologia e liberdades individuais. Tal vertente de pesquisa propõe ao estudante uma visão acerca das inovações tecnológicas, e não somente no diapasão dos desdobramentos legais, mas também nas capacidades de adaptação do profissional nessa vertente. São exemplos do retromencionado programa os cursos de Uso Cidadão da Tecnologia ${ }^{25}$ e Assistência Jurídica na Era Digital ${ }^{26}$ (HARVARD, 2018).

O Curso de Uso Cidadão da Tecnologia tem por fito explorar os esforços dos agentes públicos para a utilização da tecnologia na solução de problemas civis da comunidade. São estimuladas na disciplina as competências de criatividade e trabalho em equipe, com o objetivo de fornecer ferramentas aos alunos para que, findado o período de graduação, sejam aptos a solucionar conflitos cívico-urbanos na realidade em que se encontrarem (HARVARD, 2018).

A disciplina de Assistência e Estratégia Jurídica na Era Digital foca na reflexão e na elaboração de análise crítica e estratégica para a solução de conflitos complexos que tangenciem a seara dos desafios na vertente tecnológica enfrentados pelos empresários e pelas empresas. Por meio de abordagem multidisciplinar, explora situações de risco e negociação, bem como o estímulo ao crivo crítico de documentos, de forma a melhor desenvolver estratégias para os clientes. O curso conta com a metodologia de estudo de caso como forma primária de explanação, além da abordagem por meio de seminários (HARVARD, 2018).

Com isso, pode-se observar o empenho da instituição no desenvolvimento de competências, como o pensamento crítico, a criatividade, o trabalho em equipe, a liderança, a organização cognitiva e a solução de problemas complexos. Dessa maneira, percebe-se o alinhamento da Escola de Direito de Harvard com o ensino de habilidades pertinentes ao mercado de trabalho do século 21.

\subsection{Universidade de Stanford}

A Escola de Direito da Universidade de Stanford tem como premissa curricular a formação do operador jurídico dotado não somente das habilidades essenciais à carreira, pois busca oportunizar a customização da graduação de acordo com os interesses e ambições do aluno (UNIVERSITY OF STANFORD, 2018).

A instituição inova ao apresentar em sua matriz curricular a oferta de diversas disciplinas que promovem não somente a formação técnica do jurista, mas também as habilidades e competências para um mercado de trabalho cada vez mais dinâmico e diferenciado. Como exemplo, citam-se os cursos de Escrita Jurídica; Argumentação Oral; Direito e Criatividade; Negociação; Mediação; Desenvolvimento Profissional e Competências Gerenciais; Resolução de Problemas e Tomada de Decisão; Direito e Psicologia; Tecnologias Disruptivas. ${ }^{27}$

Em Escrita Jurídica, o discente não só aprende a desenvolver uma escrita concisa, clara e efetiva, como também a capacidade de analisar e interpretar textos. A disciplina visa a ampliar as capacidades de pesquisa, análise, interpretação, composição de textos jurídicos, síntese e edição de texto (UNIVERSITY OF STANFORD, 2018).

Em formato de oficina, a disciplina de Argumentação Oral tem por escopo desenvolver habilidades para sustentações orais, de modo que permite ao aluno refinar sua capacidade de falar em público e desenvolver a autoconfiança, bem como sua forma de reagir prontamente às situações apresentadas (UNIVERSITY OF STANFORD, 2018).

A capacidade de pensamento criativo é explorada pela matriz curricular do curso de Direito da Universidade de Stanford, de maneira direta, pela disciplina de Direito e Criatividade, cujo escopo é diminuir a distância entre Direito e Arte, de modo que permite o aprimoramento da capacidade de pensar criativamente e o uso da imaginação do discente na solução de problemas complexos. Para tanto, inicialmente,

\footnotetext{
Traduziu-se: City Use of Technology.

Traduziu-se: Counseling and Legal Strategy in the Digital Age.

No original: "Legal Writing; Oral Argument.; Law and Creativity; Negotiation; Mediation.; Problem Solving and Decision Making; Law and Psychology; Disruptive Technologies: their impact in our laws and the laws' impact on the technology".
} 
são discutidas várias formas de mídia (filmes, livros, seriados) e quais tangências com a ciência do Direito podem ser extraídas. Em seguida, os alunos devem trabalhar a escrita criativa por meio da elaboração e análise de textos para discussão em grupo (UNIVERSITY OF STANFORD, 2018).

As disciplinas de Negociação, Mediação e Resolução de Problemas e Tomada de Decisão correspondem ao modo como a instituição em questão estimula no discente a capacidade de resolução de conflitos complexos pelas vias alternativas, bem como o gerenciamento de suas atividades como jurista.

Assim, desenvolve competências que ampliam sua compreensão das situações de negociação, bem como fornece ferramentas e conceitos para preparar e criar formas diversas de resolução de problemas, isto é, extrair da experiência novos conhecimentos. Além do pensamento de evitar o litígio, após o curso, os alunos conseguem compreender as naturezas dos conflitos e quais são os princípios para o seu gerenciamento (UNIVERSITY OF STANFORD, 2018).

Tecnologias disruptivas são aquelas que dão origem a novos mercados e formas de negociação (CHRISTENSEN, 1997). Nesse sentido, observa-se que o operador jurídico deve atentar para essas inovações, uma vez que muitas delas vão de encontro a regulamentações, políticas e leis existentes para a manutenção de um status quo.

Nessa mesma linha de pensamento, a Universidade de Stanford fornece a possibilidade de o discente ampliar seus conhecimentos nessa seara, de modo que possa não somente ajudar seus clientes a se manterem no mercado, como possa ajudá-los a prosperar. Para tanto, tem-se a disciplina de Tecnologias Disruptivas, pela qual se gera a discussão sobre temáticas de uso de inteligências artificiais, como carros que não necessitam de motoristas, além de examinar os desafios legais e as oportunidades apresentadas por essas tecnologias (UNIVERSITY OF STANFORD, 2018).

Mediante a explanação de algumas das disciplinas ofertadas em seu currículo, percebe-se a relevância que a instituição em questão preza, não somente pelo desenvolvimento de aptidões, tais como o pensamento criativo, a comunicação e a empatia, mas também pela preocupação com o posicionamento do estudante em período após a graduação, uma vez que se mostra atenta às maneiras de prepará-lo para o mercado de trabalho jurídico em face das necessidades do século XXI.

\section{Conclusão}

Na perspectiva da análise dos estudos sobre as habilidades necessárias aos profissionais do século XXI e dos impactos causados pela tecnologia no mercado de trabalho, em especial, no setor jurídico, aponta-se para a necessidade do desenvolvimento e do aprimoramento dessas habilidades, especialmente no tocante ao que a tecnologia é incapaz de promover.

No âmbito jurídico, especificamente, o surgimento das legaltechs propiciou ferramentas tecnológicas capazes de suprir a atividade humana de forma mais célere e eficaz, tais como a análise e a coleta de dados, as jurisprudências, a redação de textos jurídicos (a exemplo de contratos, petições e acordos societários), assim como a possibilidade de narrativa de experiências judiciais por meio de fóruns online.

Desse modo, conclui-se, de acordo com as pesquisas e experiências apresentadas, que a profissão jurídica passa por mudanças significativas, requerendo novas competências para o profissional do Direito, tais como: a capacidade de trabalho em equipe, pensamento crítico para resolução de problemas, liderança, criatividade e habilidades de comunicação. Portanto, a necessidade de pessoas capazes de prevenir e solucionar conflitos de forma eficiente, aptas à criação de estratégias e dotadas de uma visão sistêmica.

Em decorrência disso, novas metodologias e temáticas estão sendo desenvolvidas em universidades, como Harvard e Stanfford, apontando a formação jurídica para uma nova direção, bem além da formação técnica, com forte foco nas habilidades citadas.

Isto posto, faz-se relevante observar a atualização do modelo tradicional de ensino jurídico, de forma a visar uma modificação das matrizes curriculares, dos critérios de avaliação e da aplicação dos métodos de lecionar, para que estejam em consonância com as necessidades dos profissionais do século XXI. 


\section{Referências}

ACCENTURE. 2013 Accenture Skills and Employment Trends Survey: Perspectives on the Perspectives on Training. Dublin, 2013. Disponível em: <https://www.accenture.com/sk-en/ /media/ Accenture/Conversion-Assets/DotCom/Documents/Global/PDF/Strategy_3/Accenture-2013-Skills-AndEmployment-Trends-Survey-Perspectives-On-Training.pdf>. Acesso em: 31 jan. 2018.

ALTMAN WEIL. Law firms in transition - an Altman Weil Flash Survey. Newtown Square: Altman Weil, 2015. Disponível em: <http://www.altmanweil.com/dir_docs/resource/1c789ef2-5cff-463a-863a2248d23882a7_document.pdf>. Acesso em: 31 jan. 2018.

AMERICAN MANAGMENT ASSOCIATION; PARTNERSHIP FOR 21ST CENTURY SKILLS. Critical Skills Survey, AMA, 2010. Disponível em:<http://www.p21.org/storage/documents/Critical\%20Skills\%20 Survey\%20Executive\%20Summary.pdf>. Acesso em: 31 jan. 2018.

BALCAR, J. Soft skills and their wage returns: overview of empirical literature. Review of Economic Perspectives, Národhospodarsky Obzor, v. 14, n. 1, p. 3-15, 2014.

CARNEVALE, A. 21st century competencies for college and career readiness. Broken Arrow: National Career Development Association, 2013. p. 1-9. Disponível em:<https://1gyhoq479ufd3yna29x7 ubjn-wpengine.netdna-ssl.com/wp-content/uploads/21stCenturyCompetencies.pdf $>$. Acesso em: 31 jan. 2018.

CHRISTENSEN, Clayton. The innovator's dilemma. Massachusetts: Harvard Business School Press, 1997.

COLINS, Julia. Possible futures: thoughts on what awaits HLS and the legal profession. Harvard Law Bulletin, fall 2017. Disponível em: <https://today.law.harvard.edu/feature/possible-futures/>. Acesso em: 31 jan. 2018.

EGER, H.; GROSSMANN, V. Noncognitive abilities and within-group wage inequality. Bonn: Institute for the Study of Labour, 2004.

HARVARD UNIVERSITY. Analytica Methods for Lawyers. Course Catalog. 2018. Disponível em: <http:// hls.harvard.edu/academics/curriculum/catalog/default.aspx?o=69487>. Acesso em: 2 fev. 2018.

HARVARD UNIVERSITY. Business Strategies for Lawyers. Course Catalog. 2018. Disponível em: <http://hls.harvard.edu/academics/curriculum/catalog/default.aspx?o=69488>. Acesso em: 2 fev. 2018.

HARVARD UNIVERSITY. City Use of Technology. Course Catalog. 2018. Disponível em: < http://hls. harvard.edu/academics/curriculum/catalog/default.aspx?o=69100>. Acesso em: 2 fev. 2018.

HARVARD UNIVERSITY. Counseling and Legal Strategy in the Digital Age. Course Catalog. 2018. Disponível em: <http://hls.harvard.edu/academics/curriculum/catalog/default.aspx?o=68060>. Acesso em: 2 fev. 2018.

HARVARD UNIVERSITY. First-Year legal research and writing program, 2018. Disponível em: <http:// hls.harvard.edu/dept//rw/course-overview/>. Acesso em: 1 fev. 2018.

HARVARD UNIVERSITY. J. D. Program - The fisrt year, 2018. Disponível em: <http://hls.harvard.edu/ dept/academics/degree-programs/j-d-program/>. Acesso em: 31 jan. 2018.

HARVARD UNIVERSITY. J. D. Program - The Upper Level Years, 2018. Disponível em: <http://hls. harvard.edu/dept/academics/degree-programs/j-d-program/>. Acesso em: 1 fev. 2018.

HARVARD UNIVERSITY. Academic offerings. Law, Science and Technology, 2018. Disponível em: $<$ http://hls.harvard.edu/dept/academics/programs-of-study/law-science-and-technology/academicofferings-law-science-and-technology/>>. Acesso em: 2 fev. 2018.

HARVARD UNIVERSITY. Leading innovative ventures, 2018. Disponível em: <http://www.hbs.edu/ coursecatalog/2030.html>. Acesso em: 2 fev. 2018.

HARVARD UNIVERSITY. Problem solving workshop, 2018. Disponível em: <http://hls.harvard.edu/ dept/academics/winter-term/problem-solving-workshop/>. Acesso em: 1 fev. 2018. 
HARVARD UNIVERSITY. Programs of Studying, 2018. Disponível em: <http://hls.harvard.edu/dept/ academics/programs-of-study/>. Acesso em: 2 fev. 2018.

INTERNATIONAL LABOUR ORGANIZATION. Skills for improved productivity, employment growth and development. Trabalho apresentado no International Labour Conference. Geneva. 2008. Disponível em: <https://www.ilo.org/wcmsp5/groups/public/---ed_norm/---relconf/documents/meetingdocument/ wcms_092054.pdf>. Acesso em: 2 fev. 2018.

MANPOWER GROUP. WORLD ECONOMIC FORUM. New Vision for Education - Unlocking the Potential of Technology, 2015. Disponível em:<http://www3.weforum.org/docs/WEFUSA_ NewVisionforEducation_Report2015.pdf>. Acesso em: 2 fev. 2018.

MCKINSEY GLOBAL INSTITUTE. Jobs lost, jobs gained: workforce transitions in a time of automation. 2017. Disponível em: < https://www.mckinsey.com/ /media/McKinsey/Global\%20Themes/Future\%20 of $\% 20$ Organizations/What $\% 20$ the $\% 20$ future $\% 20$ of $\% 2$ work $\% 20$ will\%20mean $\% 20$ for $\% 20$ jobs $\% 20$ skills\%20and\%20wages/MGI-Jobs-Lost-Jobs-Gained-Report-December-6-2017.ashx>. Acesso em: 09 maio 2018.

PRADUROUX, Sabrina; PAIVA, Valeria de; CARO, Luigi di. Legal Tech Start-ups: State of the Art and Trends, 2016. Disponível em: <http://vcvpaiva.github.io/includes/pubs/2016-legal.pdf>. Acesso em: 31 jan. 2018.

ROSS. What is Ross? 2018. Disponível em: <https://rossintelligence.com/ross/>. Acesso em: 31 jan. 2018.

SALOMÃO, Celina. Lawtechs: tecnologia transformando os negócios do Direito. Falando de TI, 2017. Disponivel em: < https://www.ibm.com/blogs/robertoa/2017/05/lawtechs-a-tecnologia-transformando-osnegocios-do-direito/>. Acesso em: 31 jan. 2018.

SCHWAB, Klaus. A quarta revolução industrial. São Paulo: Edipro, 2016.

SPERANDIO, Henrique Raimundo do Carmo. Desafios jurídicos frente às novas tecnologias: a inteligência artificial e seus impactos na área jurídica. São Paulo: FGV, 2016.

SUSSKIND, Richard. Tomorrow's Lawyers. Nova York: Oxford University Press, 2013.

SUSSKIND, Richard; SUSSKIND, Daniel. The future of professions: how technology will transform the work of human experts. New York: Oxford, 2017.

UNIVERSITY OF STANFORD. Disruptive Technologies: their impact in our laws and the laws' impact on the technology. Course Catalog, 2018. Disponível em: <https://law.stanford.edu/courses/disruptivetechnologies-their-impact-on-our-laws-and-the-laws-impact-on-the-technology/>. Acesso em: 2 fev. 2018.

UNIVERSITY OF STANFORD. Advanced Legal Writing. Course Catalog., 2018. Disponível em: < https:// law.stanford.edu/courses/advanced-legal-writing-business-transactions/>. Acesso em: 2 fev. 2018.

UNIVERSITY OF STANFORD. Build a foundation that will support your aspirations, 2018.

Disponível em: <https://law.stanford.edu/education/courses/\#slsnav-first-year-academics>. Acesso em: 2 fev. 2018.

UNIVERSITY OF STANFORD. Law and Creativity. Course Catalog., 2018. Disponível em: <https://law. stanford.edu/courses/writing-workshop-law-and-creativity/l>. Acesso em: 2 fev. 2018.

UNIVERSITY OF STANFORD. Law and Psychology. Course Catalog., 2018. Disponível em: <https:// law.stanford.edu/courses/law-and-psychology/>. Acesso em: 2 fev. 2018.

UNIVERSITY OF STANFORD. Negotiation; mediation; problem solving and decision making. Course Catalog, 2018. Disponível em: <https://law.stanford.edu/courses/negotiation//>. Acesso em: 2 fev. 2018.

UNIVERSITY OF STANFORD. Oral argument workshop. Course Catalog., 2018. Disponível em: <https://law.stanford.edu/courses/oral-argument-workshop//>. Acesso em: 2 fev. 2018.

VERJEIJL, Bart et al. New breakthroughs in computational law - Code X Future Law Conference. Stanford University. Disponível em: <https://www.youtube.com/watch?v=efr9VctcMe8>. Acesso em: 30 jan. 2018. 
WORLD ECONOMIC FORUM - WEF. 6 quotes from Davos on the future of education, 2018. Disponível em: <https://www.weforum.org/agenda/2018/01/top-quotes-from-davos-on-the-future-ofeducation>. Acesso em: 30 jan. 2018.

WORLD ECONOMIC FORUM - WEF. Future of Jobs report, 2016. Disponível em: <http://www3. weforum.org/docs/WEF_Future_of_Jobs.pdf>. Acesso em: 25 dez. 2017.

WORLD ECONOMIC FORUM. New vision for education - Unlocking the potential of technology, 2015. Disponível em: <https://widgets.weforum.org/nve-2015/chapter1.html>. Acesso em: 29 jan. 2018.

Recebido em: 01/06/2018

Aprovado em: 25/06/2018 